\title{
USING BEM TO PREDICT THE EFFECTIVE THERMAL CONDUCTIVITY FOR HETEROGENEOUS MATERIALS
}

\author{
M. B. A. M. Oberg, \\ C. T. M. Anflor, \\ and J. N. V. Goulart \\ Universidade de Brasília \\ Grupo de Mecânica Computacional e \\ Experimental (GMEC) Campus FGA \\ Área Especial, Projeção A, Setor Leste \\ CEP 72405-610, Gama, Distrito Federal, Brasil \\ anflor@unb.br \\ Received: March 04, 2015 \\ Revised: April 06, 2015 \\ Accepted: May 07, 2015 \\ ABSTRACT \\ the result. \\ conductivity

\section{NOMENCLATURE} \\ A coefficient matrix \\ $\mathrm{B}$ right-hand side vector \\ C shape factor \\ d diameter of the inclusions, $\mathrm{mm}$ \\ $\mathrm{G} \quad$ boundary elements $\mathrm{G}$ matrix \\ $\mathrm{H}$ boundary elements $\mathrm{H}$ matrix \\ $\mathrm{K}$ thermal conductivity coefficient, W/m.K \\ $\mathrm{L}$ distance between cooled and heated edges, $\mathrm{mm}$ \\ $\mathrm{n} \quad$ number of inclusions inserted \\ $\mathrm{NE}$ number of elements \\ q heat flux, $\mathrm{W} / \mathrm{m}^{2}$ \\ $\mathrm{r}$ distance between collocation and field points \\ $\mathrm{R}$ percentage of area occupied by the inclusions \\ $\mathrm{T}$ prescribed temperature \\ $\mathrm{u}$ temperature, $\mathrm{K}$
}

This work presents a study on the effective thermal conductivity in material with heterogeneous composition in two dimensions. The Boundary Elements Method (BEM) is used to solve the steady state potential equations. The sub regions technique was implemented in order to take into account the effects of these inclusions inside the domain. In the numerical implementation, the inclusions are randomly generated in a Representative Volume Element (RVE) domain. The Average Field Theory is used to predict the effective properties (macroscopic) of the material with heterogeneous composition. The material is characterized by a specified volume fraction as well as the inclusion's size. The samples are composed of square domains with defined number of randomly distributed inclusions and submitted to a condition of unidirectional heat conduction. Each set of samples is analyzed several times in order to guarantee statistical stability of

Keywords: boundary elements, representative volume element, thermal

\section{Greek symbols}

$\Gamma \quad$ boundary of the domain

$\eta \quad$ outward normal

$\lambda \quad$ unknown vector

$\Omega \quad$ domain

\section{Subscripts}

$$
\begin{array}{ll}
\text { c } & \text { cooled edge } \\
\text { eff } & \text { effective } \\
\text { h } & \text { heated edge }
\end{array}
$$

\section{Superscripts}

\footnotetext{
* fundamental solution

- $\quad$ prescribed boundary condition
}

\section{INTRODUCTION}

The technological advances in recent times reveal an increasing demand for advanced materials that provide high performance and efficiency. The development of optimized "tailor-made" materials for specific applications, which may require certain values of determined mechanical properties, has been quite a requested field of research lately.

Samantray et al. (2006) present a study on the development of correlational relations to determine the effective thermal conductivity of two-phase materials. Based on experimental data, it succeeded in developing correlational relations for three specific two-phased problems. This work points out the need for more accurate and efficient methods for prediction of the thermal properties of heterogeneous composition materials.

Wang and Pan (2008) evince that most of the available methods for the prediction of material properties are based on idealized periodic constructions. Therefore, it proposes a computational modelling of open-cell foams with random cell generation and growth, making possible the analysis of more complex and realistic porous structures.

At a microstructural level, materials are typically heterogeneous with many possible constitutional elements, each with its own physical properties. Starting at a microstructural observation scale, a volume element contains just a few microstructural components, therefore the effective properties of this element are largely influenced by the properties of the individual microstructural components within it. When increased the observation scale, approaching to a macrostructural 
scale, the influence of a single microstructural component over the volume element effective properties is reduced, as more components now compose it. In this way, as the observational scale is increased, increasing the number of microstructural components in the observed volume element, it is also noticed a variation of its effective properties. This behavior persists until a certain scale is reached and the observed effective properties approximate the material's macroscopic properties. In Terada and Kikuchi (1996) it is proposed a homogenization method of materials with heterogeneous composition, the representative volume element method. It consists in determining a characteristic length for the observed volume element at which the effective properties measured over the volume element boundaries approach the macroscopic properties of the material. This volume element with the characteristic length is named as the representative volume element (RVE).

An industrial application of the effective properties concept is presented in Kwon et al. (2009). This work proposes a study of the effective thermal conductivities of porous materials applied on the fabrication of Vacuum Insulation Panels (VIPs) using simplified cell models. Also considering other heat transfer mechanisms, such as gaseous and radiative conductivities, the elaborated mathematical models provided relatively accurate results compared to experimental data for every studied type of material, except for powders. This evinces the limitation of simplified models and the need for other methods for modeling materials with complex microstructures.

The boundary elements method (BEM) represents an efficient tool for the modelling of linear problems such as heat transfer by conduction or even the mechanical behavior of materials in elastic regimen. It is based on integrals evaluated over the boundaries, reducing the discretization process only to the domain's boundaries. In this sense, a reduced number of elements are required to the problem in comparison with other methods such as the finite element method (FEM), which requires the whole domain to be discretized. Due to its ability to analyze problems considering only information over the domain's boundaries, the BEM has a natural compatibility with the RVE method.

This natural compatibility is explored in Buroni and Marczak (2008) and Zarichta (2008) where both methods, the BEM and RVE, are brought together to elaborate methodologies for the prediction of effective properties of micro-porous materials. The first one, Buroni and Marczak (2008), focuses in the elastic effective properties of isotropic and orthotropic porous materials determining the RVE conditions through statistical analysis. In the same way, Zarichta (2008) applies a similar methodology now turned to the analysis of permanent potential problems and the determination of the effective thermal conductivity coefficient for micro-porous materials.

Dondero et al. (2011) present a modelling of a bidimensional heat transfer problem of plate with several random generated holes to simulate a RVE condition for a micro porous material. The numerical analysis proceeds using a "Fast Multipole" BEM formulation for increased computational efficiency. This work also presents an experimental analysis based on thermo graphical imaging in order to validate the obtained numerical results. The confrontation between numerical and experimental results showed a difference below 5\%, indicating a good performance of the presented predictive methodology.

Instead of micro-porosity, this work presents a RVE study of the effective thermal conductivity property of materials composed by two different micro-constituents with different overall properties. The study bases itself on a numerical analysis using BEM with a formulation that considers the intersection between two different sub-regions representing the micro-constituents that compose the domain.

\section{THEORY}

\section{BEM formulation for potential heat transfer}

The modelling of potential heat transfer problems in boundary elements is, perhaps, the most widely diffused BEM modelling case, and its complete detailed elaboration can be found in many books, as Wrobel (2002) and Kane (1994).

$$
\nabla^{2} u(x)=0 \quad \forall x \in \Omega
$$

A brief review on the boundary element method using constant elements is summarized in this work. An initial domain depicted in Fig. 1 is established with boundary conditions prescribed on its boundary. Considering a Laplace equation governing a 2D potential problems presented as Eq. (1).

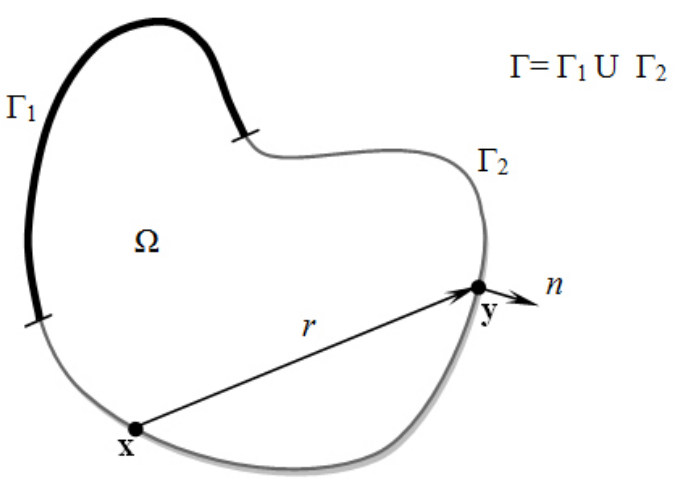

Figure 1. Domain and $\Omega$ its boundary $\Gamma$.

For a potential problem, three kinds of boundary 
conditions may be imposed: Dirichlet, Neumann and/or Robin. For this presentation, the first and second boundary conditions are imposed as:

$$
\begin{array}{ll}
u(x)=\bar{u}(x) ; & \forall x \in \Gamma_{1} \\
q(x)=\frac{\partial u}{\partial \eta}(x)=\bar{q}(x) & \forall x \in \Gamma_{2}
\end{array}
$$

where $u$ is the potential field in domain $\Omega, \Gamma$ is the boundary of $\Omega, \mathrm{n}$ is the outward normal. Note that the barred quantities are the values imposed by the boundary conditions. The solution of Eq. (2) under boundary conditions is given by:

$$
\begin{gathered}
u^{*}(x, y)=\frac{1}{2 \pi} \ln (r) \\
q^{*}(x, y)=\frac{\partial u^{*}(x, y)}{\partial \eta(y)}=\frac{1}{2 \pi r} \frac{\partial r}{\partial \eta}
\end{gathered}
$$

where $u^{*}(x, y)$ and $q^{*}(x, y)$ are the Green's functions for $2 \mathrm{D}$ problems. And $r$ represents the distance between the collocation point $\mathrm{x}$ and the field point $\mathrm{y}$, as depicted in Fig. 1. Taking $\mathrm{x}$ to the boundary, the classic boundary integral equation (BIE) formulation of BEM is obtained $\forall x \in \Omega$ :

$$
C(x) u(x)=\int_{\Gamma} u^{*}(x, y) q(y)-q^{*}(x, y) u(y) d \Gamma(y)
$$

If the boundary is smooth at the collocation point $\mathrm{x}$, the coefficient $\mathrm{C}(\mathrm{x})=1 / 2$. The next step consists in discretizing the boundary $\Gamma$ using $N$ constant elements. The discretized equation of BIE is now presented as:

$$
\frac{1}{2} u_{i}=\sum_{j=1}^{N} G_{i j} q_{j}-\sum_{j=1}^{N} H_{i j} u_{j} \quad i=1,2,3, \ldots, N
$$

where the and $(j=1,2, \ldots, N)$ are the nodal values of the $\mathrm{u}$ and $\mathrm{q}$ at the element $\Delta \Gamma \mathrm{j}$, respectively. Applying the boundary conditions at each node and switching the columns for grouping the unknown variables, one finds:

$$
A \lambda=B
$$

where $\mathrm{A}$ is the coefficient matrix, $\lambda$ the unknown vector and $\mathrm{B}$ the known right-hand side vector.

\section{BEM with sub-regions}

Specifically for this case, the sub-regions are delimited by a closed boundary region placed inside a main domain, as shown in Fig 2. The boundary elements applied to discretize the sub-region are shared with the main domain. For a better illustration of the problem, it is assumed a simpler case, as shown in Fig. 3, where two different regions have only one element in common. The sub-region $\Omega$ ' is placed in the interior of $\Omega$. The elements applied for the discretization of the inner boundary are common to both $\Omega$ ' and $\Omega$. Each of these common elements adds two new unknown variables to the linear system. To deal with these added unknown variables and couple the related regions it is necessary to impose a pair of equations that enforce the continuity condition. Therefore, for each shared elements:

$$
\begin{aligned}
& u-u^{\prime}=0 \\
& q+q^{\prime}=0
\end{aligned}
$$

where the variables $u$ and $q$ are the variables of the shared element related to one region and $u$ ' and $q$ ' are the same variables related to the equivalent element in the second sub-region.

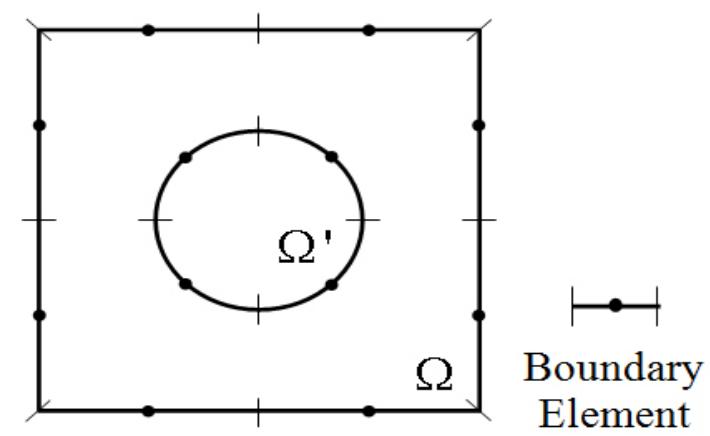

Figure 2. Example of a problem of sub-regions.

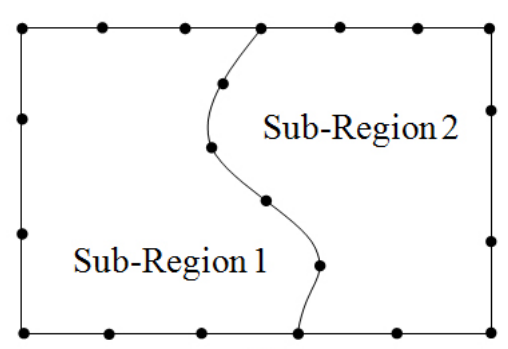

(a)

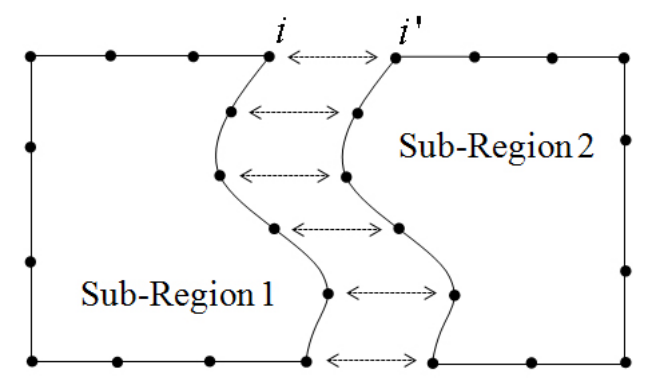

(b)

Figure 3. Sub-regions problem dealt as separated regions coupled by the continuity equations. The elements $i$ and $i$ ' are coincident. 


\section{Effective thermal conductivity using BEM}

Following a similar approach used in Zarichta (2008) for the determination of the effective thermal conductivity, it is supposed a unidirectional heat conduction problem in a square domain as illustrated in Fig. 4. To achieve the unidirectional heat flux condition the top and bottom edges are considered insulated and one of the remaining edges is uniformly heated as the other is uniformly cooled. The heated and cooled edges boundary conditions are achieved by imposing prescribed temperatures.

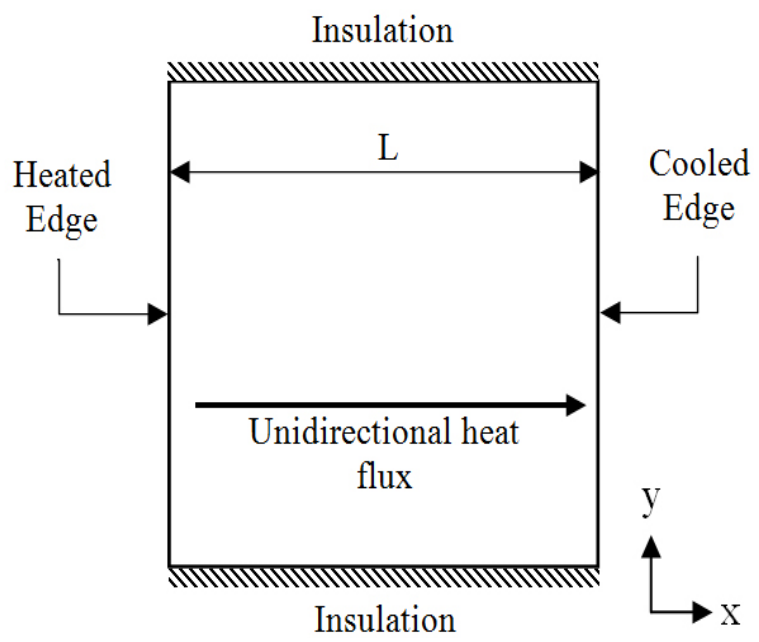

Figure 4. Scheme of the unidirectional heat flux problem on a square domain.

For the presented case, the thermal effective conductivity in the $\mathrm{x}$ direction can be calculated through the following equation, also presented in Zarichta (2008) and Wang and Pan (2008):

$$
K_{e f f}=\frac{q \cdot L}{\left(T_{h}-T_{c}\right)}
$$

where $\mathrm{q}$ represents the heat flux per area unity between the non-insulated edges, and are the temperatures along the heated and cooled edges respectively, and $\mathrm{L}$ is the distance between these two edges.

Equation (8) considers a condition of constant heat flux along the " $y$ " axis, which is no longer truth when inclusions of different materials become present inside the domain. These inclusions may be more or less conductive than the material that composes the rest of the domain, disturbing the originally unidirectional heat flux. In order to take into account this phenomenon, the effective thermal conductivity is calculated considering the sum of the individual contributions of every single element used to discretize one of the non-insulated edges. Therefore, considering a modelling with constant boundary elements, the Eq.(8) is rewritten as:

$$
K_{e f f}=\sum_{i=1}^{N E}\left(\frac{L}{N E}\right) \frac{q_{i}}{\left(T_{h_{i}}-T_{c_{i}}\right)}
$$

In Eq. (9), “ $q_{i}$ ” represents the calculated heat flux in the " $i$-th" element on one of the non-insulated edges. " $T_{h_{i}}$ " is the temperature calculated on the " $i$ - $t h$ " element on the heated edge and " $T_{c_{i}}$ " is the temperature calculated on the element that matches its " $y$ " coordinate value on the opposed edge. Last, " $N E$ " is the number of elements used to discretize one of the edges of the square domain.

\section{RVE modelling and determination}

When macroscopically observed, some materials, such as steel, present predominantly isotropic properties. However, if we reduce the observational scale to a microscopic level, the sample homogeneity and the isotropic behavior tend to disappear. In this scale, the sample has a typically heterogeneous nature, greatly influenced by its microstructural composition. The geometry, the positioning and the frequency of the present heterogeneous micro constituents are directly related to the observed domain's effective properties. For smaller observational scales the influence of a single micro constituent properties on the domain's overall properties is increased. This is caused by the presence of fewer micro constituents in such observed domains.

The Average Field Theory, as well known as the Representative Volume Element (RVE) consists in the search for the smallest observational scale on which the domain's effective properties of given set of samples converge to an isotropic behavior. This convergence is verified through the analysis of the dispersion of the physical effective properties of different samples on a same observational scale.

Based on Zarichta (2008) and Buroni and Marczak (2008) it is proposed a numerical modelling of a heterogeneous material composed by a main material matrix with several same-sized circular inclusions of a different material randomly distributed. To determine the RVE condition the percentage of area occupied by the inclusions, " $R$ " is kept constant while the number of present inclusions is increased. This approach promotes an effect analogous to the increase of the observational scale, as shown on Fig. 5. The value of " $\mathrm{R}$ " is defined as:

$$
R=100 \frac{n\left(\frac{\pi d^{2}}{4}\right)}{L^{2}}
$$

where $n$ stands for the number of inclusions inserted and $\mathrm{d}$ for its diameters. 


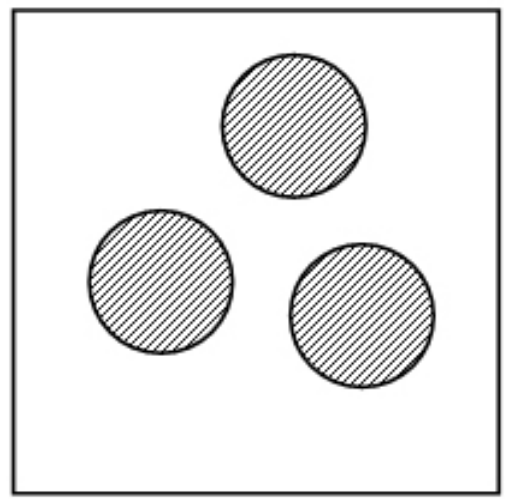

3 inclusions

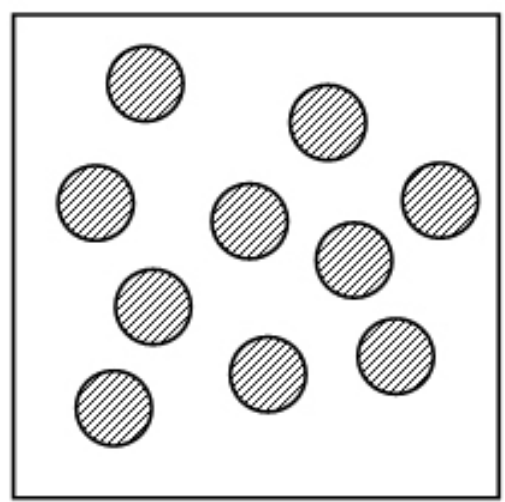

10 inclusions

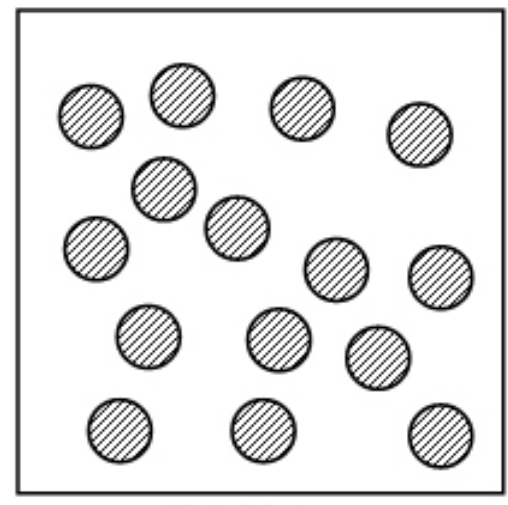

15 inclusions

Figure 5. Procedure to determinate the RVE condition.

\section{NUMERICAL RESULTS}

For this study, it was considered a hypothetical material with a thermal conductivity coefficient of $100 \mathrm{~W} / \mathrm{m} . \mathrm{K}$ with inclusions of $25 \mathrm{~W} / \mathrm{m} . \mathrm{K}$. The heated edge was kept at $100^{\circ} \mathrm{C}$ while the cooled edge at $0^{\circ}$ C. To avoid singularity issues during the positioning of the randomly generated inclusions it was set a minimum distance between two inclusions to $3 \mathrm{~mm}$. This same limit was adopted for the distances between the inclusions and the domain`s external boundaries.

The square domain outer boundaries were discretized using 22 constant boundary elements per edge, while 24 were used for each of the inclusions.

For each number of inclusions at a given fixed $\mathrm{R}$, the effective heat conductivity coefficient is calculated for different 34 samples in order to obtain a statistically consistent result and a measure of its relative dispersion. From these 34 samples a mean effective thermal conductivity is calculated to measure the convergence as more inclusions are inserted. Additionally, the standard deviation is used as a measure of the results dispersion associated to each number of inclusions.

This same analysis was repeated for $\mathrm{R}$ of: 10 , 15, 20 and $25 \%$. The results are graphically exhibited in Fig. 6. Straight lines unite the calculated mean values. Error bars represent the dispersions associated to each mean effective thermal conductivity.

From the graphical analysis, it is noticeable that for small quantities of inclusions, the calculated mean thermal conductivity values show already a relative small variation. However, it is also highlighted that fewer inclusions are associated with larger dispersion values, pointing to bigger possibility intervals. As more inclusions are present, this dispersion, given by the standard deviation, reduces. From around 37 inclusions, the variation of the dispersion associated to the increase of the number of inclusions is relatively small regardless the percentage of area occupied by the inclusions. Therefore, in face of this stabilization, the configuration with 37 can already be considered as the RVE.

The graphics exhibited on Fig. 10 depicts the influence of the percentage of area occupied by the inclusions on the effective thermal conductivity calculated for the RVE configurations obtained from the previous analysis. The alignment of the calculated points suggests a linear relation between the percentage of area occupied by the inclusions and the effective thermal conductivity of the RVEs. A similar linear relation was found in Zarichta (2008) for porous RVEs.

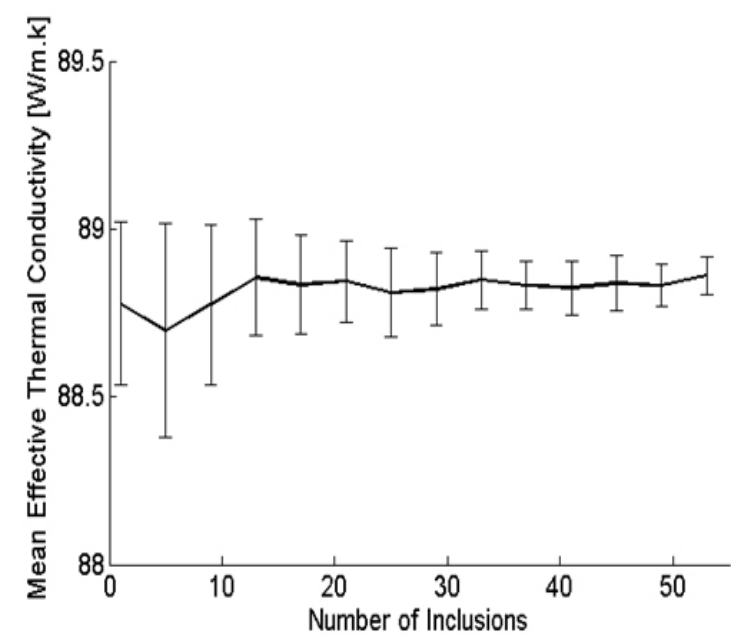

Figure 6. Mean effective thermal conductivity vs. number of inclusions inserted for: $\mathrm{R}=10 \%$. 


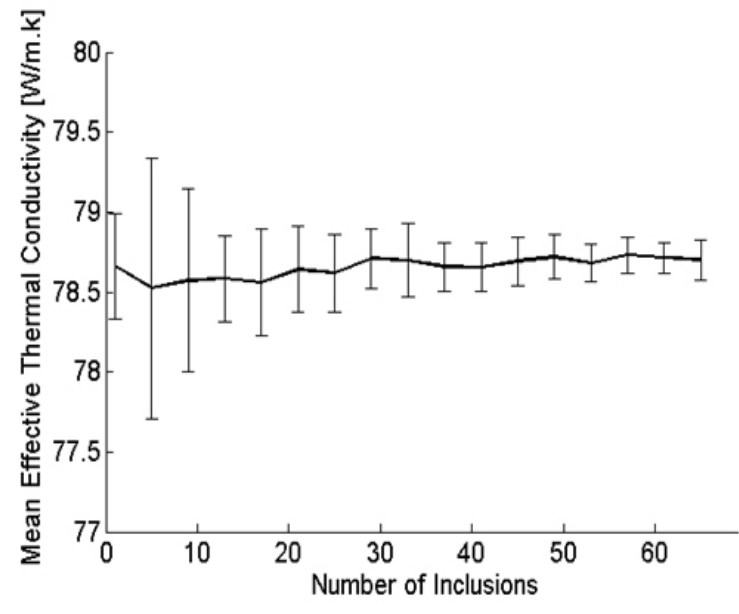

Figure 7. Mean effective thermal conductivity vs. number of inclusions inserted for: $\mathrm{R}=15 \%$.

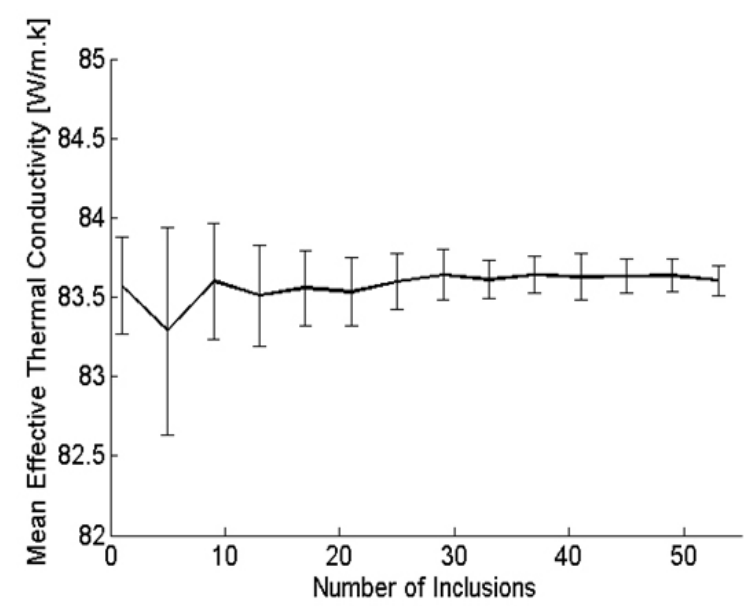

Figure 8. Mean effective thermal conductivity vs. number of inclusions inserted for: $\mathrm{R}=20 \%$.

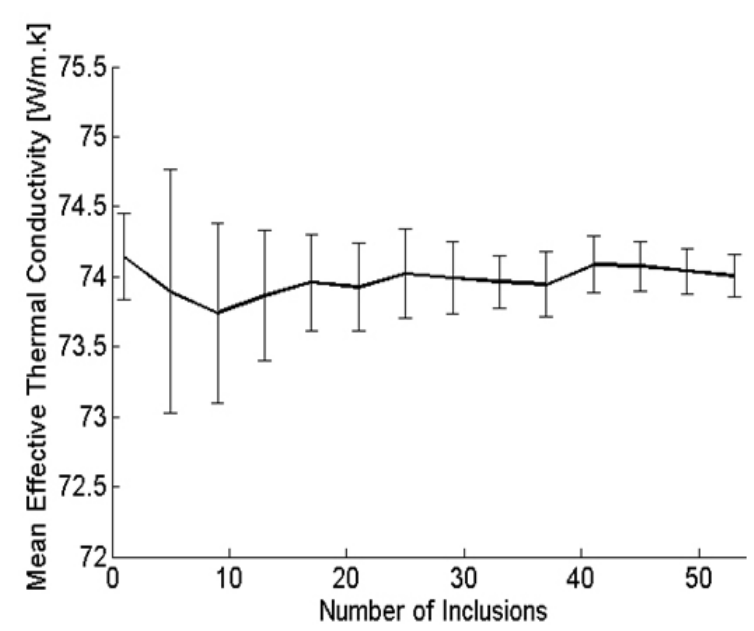

Figure 9. Mean effective thermal conductivity vs. number of inclusions inserted for: $\mathrm{R}=25 \%$.

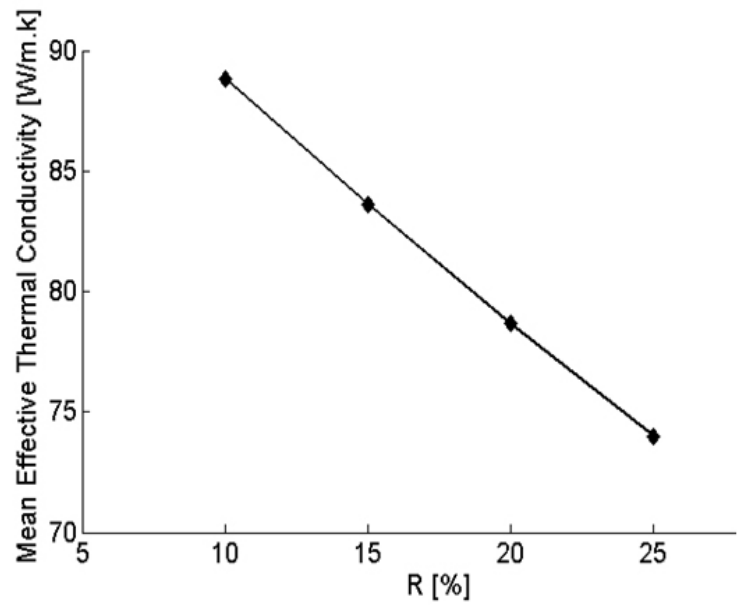

Figure 10. Mean effective thermal conductivity of the RVEs for different values of R.

\section{CONCLUSIONS}

This work presented a study on the effective thermal conductivity in materials with heterogeneous composition in two dimensions. The initial focus was on the determination of the RVE condition for materials composed by two different constituents with different thermal properties. In order to take into account the disturbance on the heat flux promoted by the presence of the material inclusions, a BEM formulation with sub-regions was used to solve the steady state potential problem. At a first moment, the percentage of area occupied by the circular inclusions was kept constant as the number of inclusions inside the domain was varied. The random positioning of the inclusions inside the domain caused a variation of the calculated effective thermal conductivity for configurations of same number of inclusions. In face of this behavior, the results were statically analyzed in order to measure its dispersion and convergence as more inclusions were inserted. This same procedure was repeated for different fractions of area occupied by the inclusions as exposed in Figs. 6 to 9. From the results of convergence, and the stabilization of its dispersion, observed in all the studied cases it was possible to conclude that a configuration of 37 inclusions can already be considered a RVE. The effective thermal conductivities obtained from the studied RVEs were graphically analyzed in Fig. 10. The observed linear behavior agreed to the similar results shown in Zarichta (2008) for porous materials, showing the feasibility of the presented methodology. The BEM formulation proved itself as a versatile and efficient tool for analysis of problems that involve sub-regions.

\section{ACKNOWLEDGEMENTS}

The authors thank CNPq for fellowships and financial support. 


\section{REFERENCES}

Buroni, F. C., and Marczak, R. J., 2008, Effective Properties of Materials with Random Micro-cavities using Special Boundary Elements, Journal of Material Science, Vol. 43, pp. 3510-3521.

Dondero, M., Cisilino, A. P., Carella, J. M., and Tomba, J. P., 2011, Effective Thermal Conductivity of Functionally Graded Random MicroHeterogeneous Materials using Representative Volume Element and BEM, International Journal of Heat and Mass Transfer, Vol. 54, pp. 3873-3881.

Kane, J. H., 1994, Boundary Element Analysis in Engineering Continuum Mechanics, Ed. Prentice Hall, New Jersey, USA, ISBN: 0-13-086927-9.

Kwon, J. S., Jang, H. C., Jung, H., and Song, T. H., 2009, Effective Thermal Conductivity of Various Filling Materials for Vacuum Insulation Panels, International Journal of Heat and Mass Transfer, Vol. 52, pp. 5525-5532.

Samantray, P. K., Karthikeyan, P., and Reddy, K. S., 2006, Estimating Effective Thermal Conductivity of Two-phase Materials, International Journal of Heat and Mass Transfer, Vol. 49, pp. 4209-4219.

Terada, K., and Kikuchi, N., 1996, Microstructural Design of Composites using the Homogenization Method and Digital Images, Materials Science Research International, Vol. 2, pp. 65-72.

Wang, M., and Pan, N., 2008, Modeling and Prediction of the Effective Thermal Conductivity of Random Open-cell Porous Foams, International Journal of Heat and Mass Transfer, Vol. 51, pp. 1325-1331.

Zarichta, C., 2008, Um Estudo Sobre Condutividade Térmica Efetiva em Materiais MicroPorosos utilizando o Método dos Elementos de Contorno, Master Thesis, Universidade Federal do Rio Grande do Sul, Porto Alegre. (in Portuguese)

Wrobel, L. C., 2002, The Boundary Element Method: Applications in Thermo-Fluids and Acoustics, Vol. 1, Wiley. 